\title{
Density Functional Study of the Electronic, Elastic, and Lattice Dynamic Properties of $\mathrm{SnS}_{2}$
}

\author{
ZHI-QIANG ZHEN ${ }^{a}$ AND HAI-YAN WANG ${ }^{b, *}$ \\ ${ }^{a}$ School of Physics and Engineering, Henan University of Science and Technology, Luoyang 471003, China \\ ${ }^{b}$ School of Materials and Engineering, Henan Polytechnic University, Jiaozuo 454003, China \\ (Received November 14, 2019; revised version January 29, 2020; in final form February 6, 2020) \\ $\mathrm{SnS}_{2}$ is an important disulfide having many excellent properties for application. Here we carried out a first- \\ principles study of its electronic, elastic and lattice dynamic properties. The band structure calculated by the \\ HSE06 functional indicates that $\mathrm{SnS}_{2}$ is an indirect band gap semiconductor with a gap of $2.16 \mathrm{eV}$. Calculated \\ partial density of states and the Mulliken charges imply that $\mathrm{SnS}_{2}$ is an ionic-covalent compound. Studies unveil \\ that $\mathrm{SnS}_{2}$ is mechanically and dynamically stable, but it is easy to crack along the $c$-axis. Phonons at its Brillouin \\ zone center were assigned by group theory analysis and their frequencies were computed. Investigation of its Born \\ effective charges and dielectric constants reveals that ions have a larger dielectric contribution than electrons in \\ the $a b$ plane, but they almost have no contribution along the $c$ direction.
}

DOI: 10.12693/APhysPolA.137.1095

PACS/topics: 62.20.de, 63.20.D-, 71.20.-b

\section{Introduction}

Layered transition metal dichalcogenides, especially their two-dimensional (2D) derivatives, have many interesting properties for possible technical applications, so they attracted the interest of researches all over the world. For example, bulk $\mathrm{MoS}_{2}$, a semiconductor with an indirect band gap of $1.29 \mathrm{eV}$, can be thinned into a $2 \mathrm{D}$ monolayer with a direct band gap of $1.90 \mathrm{eV}$ [1] accompanied by the change of the Raman scattering [2]. The monolayer has good catalytic properties [3]. Its band gap can be tuned by different methods, making it very promising for various applications [4].

As a member of layered metal dichalcogenides, hexagonal $\mathrm{SnS}_{2}$ has also attracted the interest of researches. In the experimental respect, Du et al. [5] synthesized singlecrystalline, hexagonal $\mathrm{SnS}_{2}$ nanoplatelets by solvothermal method. They found that these nanoplatelets are promising solar photocatalyst and can be used to treat waste water and contaminants. Burton et al. [6] synthesized and characterized the single-crystal $\mathrm{SnS}_{2}$ by chemical vapor transport method. Their studies reveal that $\mathrm{SnS}_{2}$ can be used as an $n$-type buffer layer in thin-film solar cells. Julien et al. [7] experimentally studied the electrical and optical properties of $\mathrm{SnS}_{2}$ by measuring its resistivity, the Hall effect, optical absorption coefficient and far-infrared spectra. Smith et al. [8] studied the Raman spectrum of $\mathrm{SnS}_{2}$ at different temperatures. They identified the short-range forces that determine the frequencies of the high-frequency optical modes using a simple force-constant model. Wang et al. [9] investigated the Raman scattering, far infrared spectrum and

*corresponding author; e-mail: haiyanwanghpu@sina.com photoluminescence of $\mathrm{SnS}_{2}$ nanocrystallites. They found that the Raman and far-infrared spectra of the nanocrystallites are slightly different from those of single crystal owing to the size effect. Bhatt et al. [10] measured the Raman spectrum of $\mathrm{SnS}_{2}$ under pressures up to $20 \mathrm{GPa}$ with temperatures up to $300 \mathrm{~K}$ and found that $\mathrm{SnS}_{2}$ is still mechanically stable. Lippens et al. [11] explained the X-ray photoelectron, emission and absorption spectroscopes by calculating the electronic structure of $\mathrm{SnS}_{2}$. Fils $\varnothing$ et al. [12] investigated the structural parameters and band structure of $\mathrm{SnS}_{2}$ in the pressure range from 0 to $20 \mathrm{GPa}$ by X-ray diffraction and density functional theory (DFT) calculations. The results indicate that the band gap of $\mathrm{SnS}_{2}$ decreases from $2.15 \mathrm{eV}$ at zero pressure to $0.88 \mathrm{eV}$ at $20 \mathrm{GPa}$, and then closes at about $33 \mathrm{GPa}$. Giberti et al. [13] synthesized nanosized $\mathrm{SnS}_{2}$ powder and characterized it. They discovered that $\mathrm{SnS}_{2}$ has a strong selectivity for the carbonyl group of aldehydes and ketones, so it can be used as gas sensor. Xiong et al. [14] fabricated $\mathrm{SnS}_{2}$ nanoflowers via a facile solvothermal process and found that they have superb selectivity to $\mathrm{NH}_{3}$. Wei et al. [15] synthesized hierarchical $\mathrm{SnS}_{2}$ nanostructures with their $\{001\}$ facets exposed having superior photocatalytic properties, which can be used to degradate methyl orange and to reduce aqueous $\mathrm{Cr}(\mathrm{VI})$. Chia et al. [16] examined the electrochemical properties of $\mathrm{SnS}_{2}$ and found that $\mathrm{SnS}_{2}$ is active in the hydrogen evolution reaction.

In the theoretical respect, He and Shen [17] studied the band structure and thermodynamic properties of $\mathrm{SnS}_{2}$. Wang et al. [18] investigated the phonon transport properties and thermal conductivity of $\mathrm{SnS}_{2}$ using first-principles calculations. Their results imply that $\mathrm{SnS}_{2}$ has a ultralow out-of-plane thermal conductivity, making it possible for use as thermoelectric material. Gonzalez and Oleynik [19] studied layer-dependent properties of $\mathrm{SnS}_{2}$ and found that the in-plane lattice 
constants, interlayer distance, and binding energy are almost layer-independent. Some research groups also studied the band structures of $\mathrm{SnS}_{2}$ nanotubes [20, 21] and electronic properties [22] and thermal conductivity [23] of $\mathrm{SnS}_{2}$ monolayer.

Although many studies were carried out on the hexagonal $\mathrm{SnS}_{2}$, its electronic, elastic and lattice dynamic properties have not been well studied. Therefore, we performed a detailed first-principles study on these above properties of $\mathrm{SnS}_{2}$ to enrich our understanding of it. The rest of this work is put in the following order: The computational methods are described in Sect. 2, the results and the related discussion are presented in Sect. 3 and the conclusions of this work are given in Sect. 4 .

\section{Computational methods}

First-principles studies were performed with the use of the CASTEP code [24]. PBE functional [25] within the generalized gradient approximation (GGA) was employed to compute the interaction energy among electrons. $\mathrm{SnS}_{2}$ is a layered material and common functionals cannot give an accurate description of its properties, so we adopted the van der Waals (vdW) correction of the Grimme type [26] with the Perdew-Burke-Ernzerhof (PBE) functional throughout this work unless explicitly stated otherwise. The on-the-fly norm-conserving pseudopotentials, generated and recommended by CASTEP for their accuracy and consistency, were used to simulate the interaction between the electrons and nuclei. The elastic constants were calculated by the stress-strain method: the strains $\varepsilon_{x x}, \varepsilon_{z z}$ and $\varepsilon_{y z}$ with the maximum amplitude of $\pm 0.3 \%$ and steps of 4 were individually applied on the crystal lattice, subsequently, the lattice was relaxed as well as the inner atomic positions to obtain the corresponding stresses, finally, the elastic constants were obtained by solving the strains-stress relations. Lattice dynamics were computed via the linear response method as implemented in CASTEP.

\section{Results and discussion}

The hexagonal $\mathrm{SnS}_{2}$ studied here has the space group of $P-3 m 1$, as illustrated in Fig. 1. The reported experimental lattice constants are $a=3.647 \AA$ and $c=5.899 \AA$ [27] and $a=3.638 \AA$ and $c=5.880 \AA$ [28]. The Wyckoff positions of the ions are: $\mathrm{Sn} 1 a(000)$ and $\mathrm{S} 2 d(1 / 3,2 / 3,0.25)$. We first tested the convergences of the lattice constants and elastic properties of $\mathrm{SnS}_{2}$ with respect to the cutoff energy and the $k$ mesh. In the geometry optimization, we used a set of strict convergence criteria: that of the energy in successive iteration is set to $5 \times 10^{-6} \mathrm{eV} /$ atom, of the forces between ions is set to $0.01 \mathrm{eV} / \AA$ and of the stresses of the lattice is set to 0.02 GPa. In calculating the elastic constants, the convergence criterion for energy is decreased to $1 \times 10^{-6} \mathrm{eV} /$ atom and that for the force is reduced to $0.002 \mathrm{eV} / \AA$. The results are displayed in Fig. 2.

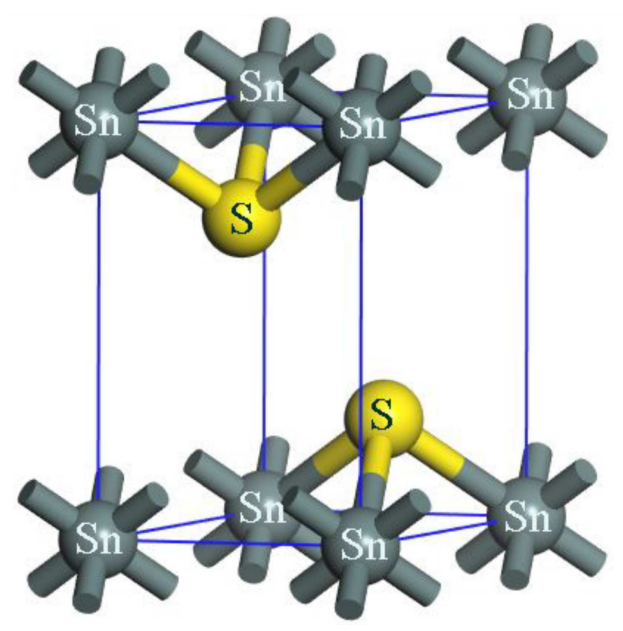

Fig. 1. Crystal structure of the hexagonal $\mathrm{SnS}_{2}$.

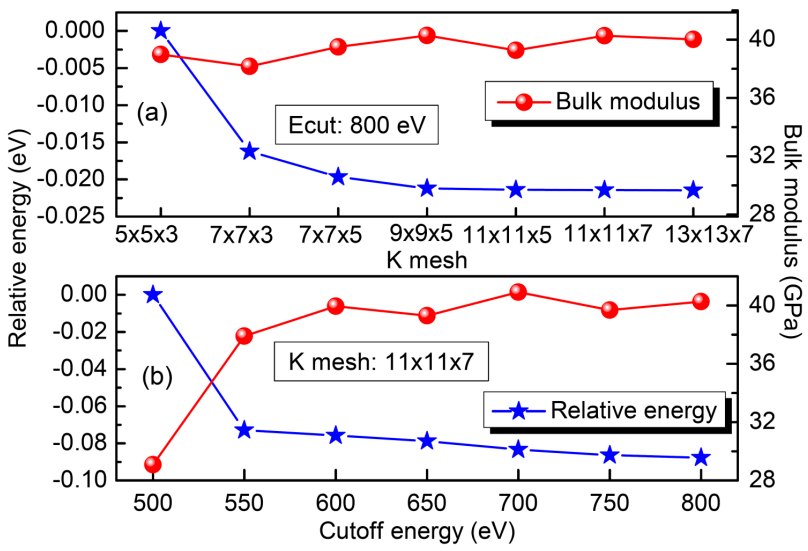

Fig. 2. (a) Relative total energy and bulk modulus as functions of $k$ mesh, the energy obtained using the $5 \times 5 \times$ 3 mesh was set to zero, and (b) relative total energy and bulk modulus as functions of cutoff energy, the energy obtained using the cutoff energy of $500 \mathrm{eV}$ was set to zero.

Fig. 2a reveals that the total energy keeps unchanged and the bulk modulus almost sticks to $40 \mathrm{GPa}$ from the $9 \times 9 \times 5$ mesh when the cutoff energy is $800 \mathrm{eV}$. Fig. $2 \mathrm{~b}$ shows that the total energy begins to converge at $550 \mathrm{eV}$ with an energy change of about $1 \mathrm{meV} /$ atom from then on when the $k$ mesh is $11 \times 11 \times 7$, which decreases to 0.4 $\mathrm{meV} /$ atom when the cutoff energy approaches $800 \mathrm{eV}$. The bulk modulus also converges to $40 \mathrm{GPa}$. The variation of the bulk modulus in these two cases should be caused by the inevitably computational error in the numerical calculations. Based on the tests, we selected the $k$ mesh of $11 \times 11 \times 7$ and the cutoff energy of $800 \mathrm{eV}$ to perform the following studies. We selected the $11 \times 11 \times 7$ mesh because it can give an almost equally spaced reciprocal space, which is expected to reduce the computational error, simultaneously, it can lessen the computational burden of the dynamic calculation. 
The lattice constants relaxed by the $\mathrm{PBE}-\mathrm{vdW}$ method are $a=3.682 \AA$ and $c=5.878 \AA$, which have relative errors of $0.95 \%(1.2 \%)$ and $-0.36 \%(0.034 \%)$, respectively, compared with the first (second) set of experimental values, which will ensure the reliability of our following studies. In addition, we also tested the pure PBE functional and other functionals provided by CASTEP with and without the vdW correction, but they give much larger relative errors than $\mathrm{PBE}-\mathrm{vdW}$, that is why we chose the PBE-vdW method in this work.

\subsection{Electronic properties}

The calculated band gap of $\mathrm{SnS}_{2}$ using the PBE-vdW method is $1.28 \mathrm{eV}$, which is smaller than the experimental values of 2.1-2.4 eV [29]. Therefore, we used the HSE06 functional [30] to calculate the electronic properties of $\mathrm{SnS}_{2}$ based on the PBE-vdW relaxed lattice. The obtained band structure along the high symmetry directions in the Brillouin zone is presented in Fig. 3a. The valence band minimum is located at the $\Gamma$ point while the conduction band maximum is located between the $\Gamma$ and $K$ points. The band gap calculated by the HSE06 functional is $2.16 \mathrm{eV}$, agreeing well with the experimental values [29]. These indicate that $\mathrm{SnS}_{2}$ is an indirect narrow-band semiconductor. The bands below the Fermi level are composed of four parts: one set of flat bands located at about $-22 \mathrm{eV}$ and three sets of dispersive bands located at around $-13 \mathrm{eV},-6 \mathrm{eV}$ and $-2.5 \mathrm{eV}$, respectively. The conduction bands seem slightly fluctuated except the lowest one. In order to explain the behavior of these bands, we computed the partial density of states of $\mathrm{SnS}_{2}$, as shown in Fig. 3b. The results reveal that the bands at about $-22 \mathrm{eV}$ originate from the $\mathrm{Sn} 4 d$ orbital, which is fully occupied by ten electrons and localized, causing the bands to be flat. Those located in the range of -15 to $-13 \mathrm{eV}$ are from the S $3 s$ and $\mathrm{Sn} 5 s$ and $5 p$ orbitals. Those located in the range of -10 to $-5 \mathrm{eV}$ come from the S $3 p$ and Sn $5 s$ orbitals. Those located in the range of $-5 \mathrm{eV}$ to the Fermi level are contributed by the $\mathrm{S} 3 p$ and Sn $5 p$ orbitals. The bands just above the Fermi level are from the Sn $5 s$ and S $3 p$ orbitals. Those in the range from 5 to $10 \mathrm{eV}$ are formed by the Sn $5 p$ and $\mathrm{S} 3 p$ orbitals. Those above are mainly from the Sn $5 s$ orbital. Obviously, the $s$ and $p$ orbitals of Sn and S overlap with each other, especially in the energy range of $-5 \mathrm{eV}$ to the Fermi level, which means that there exists hybridization between the Sn and S atoms, making the corresponding bands fluctuated. It also means that the $\mathrm{Sn}-\mathrm{S}$ bonds have some covalent property.

To further explore the bonding property of $\mathrm{SnS}_{2}$, we calculated its electron difference density and Mulliken charges using the HSE06 functional based on the PBEvdW relaxed lattice. The former is given in Fig. 4, which confirms the existence of the covalent property of the Sn-S bonds because of some electrons accumulating between the Sn and S atoms. Calculated Mulliken bond populations unveil that there are 1.09 electrons located between the bonded $\mathrm{Sn}$ and $\mathrm{S}$ atoms. The calculated

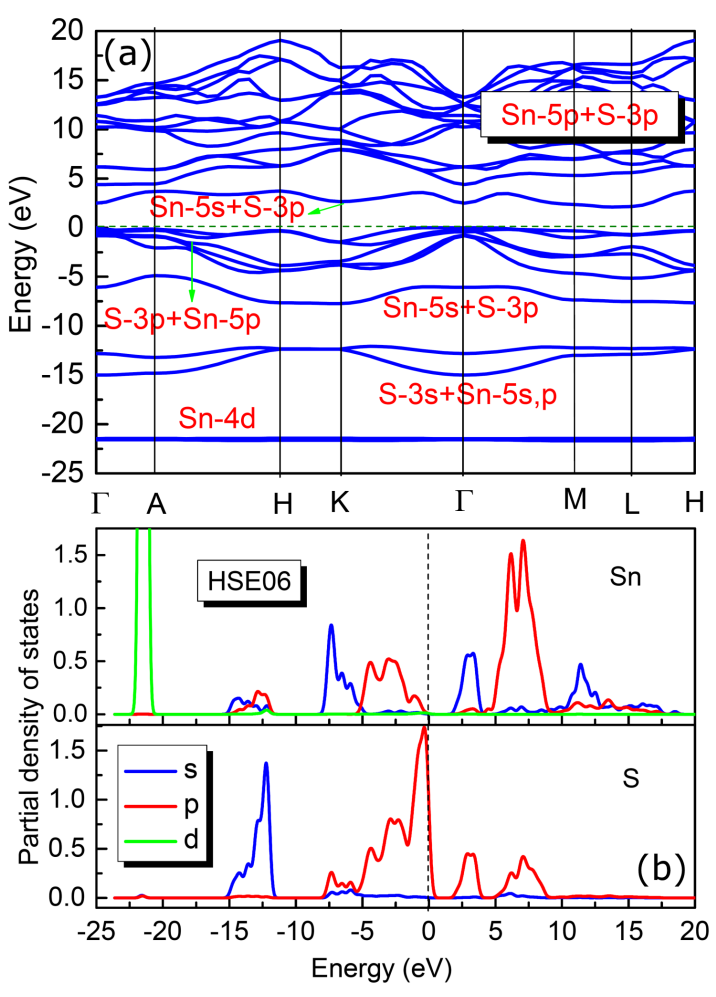

Fig. 3. Calculated (a) band structure and (b) partial density of states of $\mathrm{SnS}_{2}$ by the HSE06 functional based on the $\mathrm{PBE}-\mathrm{vdW}$ relaxed lattice.

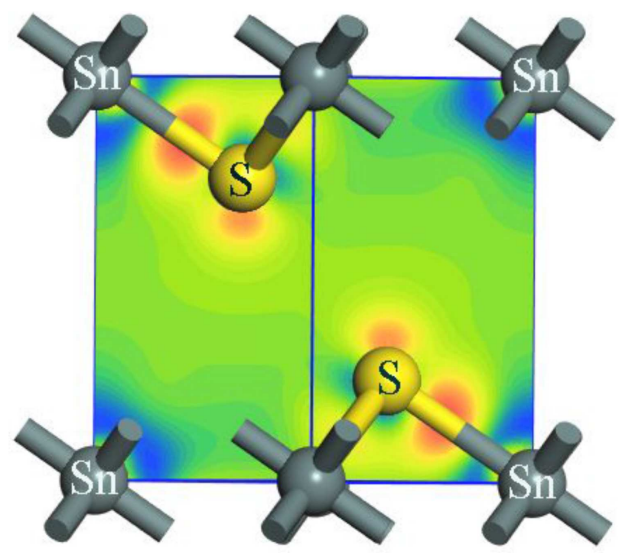

Fig. 4. Calculated electron difference density in the $(1-10)$ plane of $\mathrm{SnS}_{2}$ by the HSE06 functional based on the $\mathrm{PBE}-\mathrm{vdW}$ relaxed lattice.

atomic charges reveal that each $\mathrm{Sn}$ atom loses 0.94 electrons while each S atom gains 0.47 electrons. Therefore, $\mathrm{SnS}_{2}$ is an ionic-covalent crystal.

\subsection{Elastic properties}

Elastic constants of materials measure the stress caused by the applied strain within their elastic limit, which are important mechanical parameters reflecting their mechanical stability and stiffness. 
TABLE I

Calculated elastic constants of $\mathrm{SnS}_{2}$ by the PBE-vdW method, in GPa

\begin{tabular}{l|c|c|c|c|c|c}
\hline \hline & $C_{11}$ & $C_{33}$ & $C_{44}$ & $C_{66}$ & $C_{12}$ & $C_{13}$ \\
\hline present & 140.2 & 32.0 & 12.8 & 50.1 & 40.0 & 16.9 \\
Ref. [17] & 137.6 & 18.9 & 50.5 & 49.3 & 39.1 & 33.7
\end{tabular}

The calculated elastic constants of $\mathrm{SnS}_{2}$ are listed in Table I. For a hexagonal crystal with mechanical stability, its elastic constants should satisfy the following restrictions: $C_{44}>0, C_{11}>\left|C_{12}\right|,\left(C_{11}+C_{12}\right) C_{33}>2 C_{13}^{2}[31]$. The calculated elastic constants meet the above restrictions, so $\mathrm{SnS}_{2}$ is mechanically stable. Our calculation give much lower values of $C_{44}$ and $C_{13}$ and higher value of $C_{33}$ compared with those presented by He and Shen [17]. This should be induced by the different methods used: they used the VASP code with the PAW pseudopotentials while we employed the CASTEP code using the PBE-vdW method with the on-the-fly pseudopotentials. $C_{11}(140.2 \mathrm{GPa})$ is larger than $C_{33}(32.0 \mathrm{GPa})$, which hints that the $a / b$ axis of $\mathrm{SnS}_{2}$ is stiffer than its $c$ axis, resulting from the layered structure. $C_{12}(40.0 \mathrm{GPa})$ is significantly larger than $C_{13}(16.9 \mathrm{GPa})$, which indicates that the $c$ axis will have a larger strain than the $a / b$ axis when applying a normal stress along the $a / b$ axis. $C_{44}$ and $C_{66}$ have a close relationship with the shear deformation of crystals. For $\mathrm{SnS}_{2}, C_{44}(12.8 \mathrm{GPa})$ is considerably smaller than $C_{66}=\left(C_{11}-C_{12}\right) / 2(50.1 \mathrm{GPa})$, which indicates that $\mathrm{SnS}_{2}$ is very vulnerable to the shear deformation in the case of applying a shear stress on the $(00 \pm 1)$ planes along the $[0 \pm 10]$ directions. Under this condition, $\mathrm{SnS}_{2}$ is easy to fracture along the $c$ axis for the weak van der Waals interaction in this direction.

Because individual elastic constant of polycrystals is impossible to measure, we have to use some aggregate qualities, such as bulk modulus, shear modulus, Young's modulus and Poisson's ratio to describe their elastic properties, which can all be deduced from elastic constants as we did previously [31]. The volume change of materials under hydrostatic pressure can be weighted by the bulk modulus $B$ while their shape change can be measured by the shear modulus $G$. The computed bulk modulus of $\mathrm{SnS}_{2}$ is $40.2 \mathrm{GPa}$ and the shear modulus is predicted to be $25.3 \mathrm{GPa}$. The former is higher than the latter, which means that $\mathrm{SnS}_{2}$ has a weak ability against shear deformation and the mechanical stability of $\mathrm{SnS}_{2}$ is restricted by the shear modulus. Pugh [32] gave a criterion to classify brittle and ductile materials: A material is ductile if its $B / G$ ratio is beyond 1.75 , otherwise, it is brittle. The obtained $B / G$ ratio of $\mathrm{SnS}_{2}$ is 1.59 , so it is brittle.

Young's modulus can be used to measure the strain caused by a uniaxial stress and Poisson's ratio is employed to weight the transverse contraction strain introduced by a longitudinal stretch. The calculated Young modulus for $\mathrm{SnS}_{2}$ is $62.7 \mathrm{GPa}$. Poisson's ratio sometimes is used to judge the bonding manner within a crystal.
Haines et al. [33] pointed out that Poisson's ratio is close to 0.1 for a pure covalent crystal and is in the range of 0.2-0.3 for an ionic-covalent crystal. The Poisson ratio of $\mathrm{SnS}_{2}$ is 0.24 , which means that $\mathrm{SnS}_{2}$ is an ionic-covalent crystal.

The Debye temperature is a basic quantity having a close connection with the specific heat and melting temperature of materials. Based on the calculated elastic constants, we obtained a value of $2405.8 \mathrm{~m} / \mathrm{s}$ as the average acoustic velocity of $\mathrm{SnS}_{2}$, which finally give the value $251.6 \mathrm{~K}$ as the Debye temperature of $\mathrm{SnS}_{2}$. The low Debye temperature means a low melting temperature and thermal conductivity of $\mathrm{SnS}_{2}$.

\subsection{Lattice dynamic properties}

\subsubsection{Phonons and dispersion curves}

Phonons are induced by the collective vibration of ions within a crystal. The vibrational modes at the Brillouin zone center can be classified by group theory method. There is one chemical formula in the primitive cell of $\mathrm{SnS}_{2}$, which has three atoms, so there are nine vibrational modes at the Brillouin zone center: three are acoustic and six are optical. According to the group theory analysis, these optical modes can be divided into four irreducible components, i.e.

$$
\Gamma_{\text {opt }}=A_{1 \mathrm{~g}}(R)+A_{2 \mathrm{u}}(I)+E_{\mathrm{g}}(R)+E_{\mathrm{u}}(I) .
$$

Here the $A_{2 \mathrm{u}}$ and $E_{\mathrm{u}}$ modes are infrared-active, and the $A_{1 \mathrm{~g}}$ and $E_{\mathrm{g}}$ modes are Raman-active. To see how the constituent ions vibrate in these modes, we computed their vibrational eigenvectors and the corresponding frequencies. The results are presented in Fig. 5.

The calculated vibrational frequencies of the $E_{\mathrm{u}}, E_{\mathrm{g}}$, $A_{1 \mathrm{~g}}$, and $A_{2 \mathrm{u}}$ are $193.1,203.2,308.8$, and $327.9 \mathrm{~cm}^{-1}$, respectively, which are in accordance with the experimental values 205, 205, 315 and $340 \mathrm{~cm}^{-1}$ [8]. They also agree reasonably with the + values $198,218,309$,

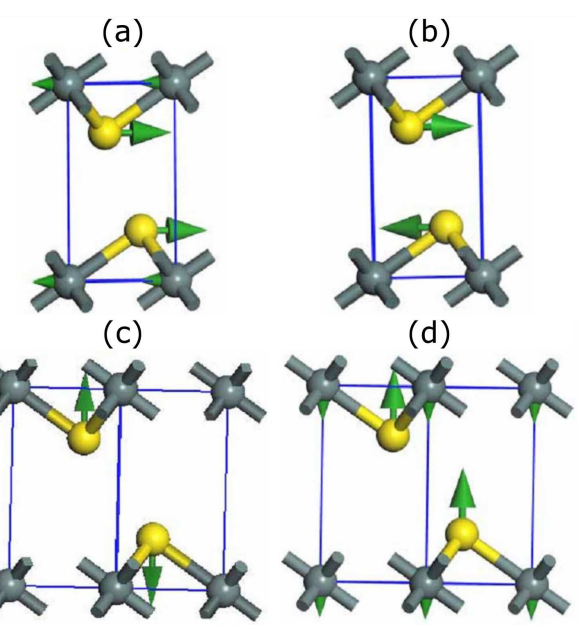

Fig. 5. Calculated vibrational eigenvectors and the corresponding frequencies (in $\mathrm{cm}^{-1}$ ) of the phonons at the Brillouin zone center of $\mathrm{SnS}_{2}$ by the PBE-vdW method. 


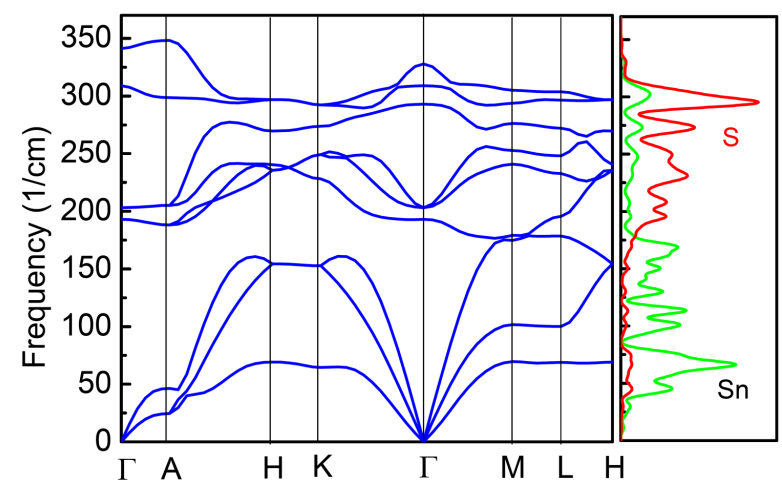

Fig. 6. Calculated phonon dispersion curves (left part) and phonon partial density of states (right part) of $\mathrm{SnS}_{2}$ by the PBE-vdW method.

and $354 \mathrm{~cm}^{-1}$ measured by Wang et al. [9]. For $\mathrm{SnS}_{2}$, the singly degenerate $A_{1 \mathrm{~g}}$ and $A_{2 \mathrm{u}}$ modes are induced by the ions oscillating along the $c$ axis, but those doubly degenerate $E_{\mathrm{u}}$ and $E_{\mathrm{g}}$ modes are caused by the ions oscillating in the $a b$ plane. In the Raman-active mode $A_{1 \mathrm{~g}}\left(E_{\mathrm{g}}\right)$, the $\mathrm{S}$ ions move out of phase in the $c(2 \boldsymbol{a}+\boldsymbol{b})$ direction with the $\mathrm{Sn}$ ions kept still. The frequency of the $A_{1 \mathrm{~g}}$ mode is higher than the $E_{\mathrm{g}}$ for the high symmetry of the three $\mathrm{Sn}-\mathrm{S}$ bonds in the former mode. In both modes, the dipole moment does not change with the vibration, but the distribution of electronic cloud changes, so they are Raman-active. In the infrared-active mode $A_{2 \mathrm{u}}\left(E_{\mathrm{u}}\right)$, these two $\mathrm{S}$ ions move in phase along the $c(2 \boldsymbol{a}+\boldsymbol{b})$ direction and the $\mathrm{Sn}$ ions vibrate oppositely. The $A_{2 \mathrm{u}}$ mode has a higher frequency than the $E_{\mathrm{u}}$ mode for the same reason given above. In these two modes, the dipole moment changes with the vibration, but the distribution of the electronic cloud does not, so they are infrared-active.

To see whether the hexagonal $\mathrm{SnS}_{2}$ is dynamically stable, we further computed its phonon dispersion curves and displayed them in Fig. 6.

There are no imaginary frequencies in the phonon dispersion curves, so the hexagonal $\mathrm{SnS}_{2}$ is dynamically stable. Figure 6 shows that the acoustic and optical branches meet around the $M$ point at about $175 \mathrm{~cm}^{-1}$. To find the origin of those curves, we also computed the phonon partial density of states of $\mathrm{SnS}_{2}$, as given in the right part of Fig. 6. The result reveals that the bands below $175 \mathrm{~cm}^{-1}$ are mainly contributed by the $\mathrm{Sn}$ ions for their large atomic weight while those above come from the $\mathrm{S}$ ions for their light atomic weight.

\subsubsection{Born effective charges and dielectric properties}

The Born effective charges weight the strength of longrange Coulomb interaction between nuclei within crystals. The Born effective charge (BEC) $Z_{k, \beta \alpha}^{*}$ of the ion $k$ can be defined as the $\alpha$ component of the force $F$ induced on the ion $k$ by the $\beta$ component of the applied electric field $E$ when the ion is fixed, i.e. $Z_{k, \beta \alpha}^{*}=\left.\frac{\partial F_{k, \alpha}}{\partial E_{\beta}}\right|_{\tau=0}[34]$. Commonly, BECs of each ion in a crystal form a tensor (BECT).
Because $\mathrm{SnS}_{2}$ has high symmetries of ionic site and space group, only the three diagonal components $x x$, yy and $z z$ are nonzero, in which the $x x$ and $y y$ components are equal. Calculations reveal that the BECs of the Sn ion are $Z_{x x}^{*}=Z_{y y}^{*}=4.99$ and $Z_{z z}^{*}=1.84$. The former two are considerably larger than the latter, which implies that the interaction between the $\mathrm{Sn}$ and $\mathrm{S}$ ions is strong in the $a b$ plane, due to the strong $\mathrm{Sn}-\mathrm{S}$ bonds. The values of the $\mathrm{S}$ ion are $Z_{x x}^{*}=Z_{y y}^{*}=-2.50$ and $Z_{z z}^{*}=-0.92$, which also hints that the interaction in the $a b$ plane is stronger than that in the $c$ direction, due to the weak van der Waals along the $c$ axis. All the offdiagonal components are zero, which means that displacing the sublattices formed by the Sn or S ions individually along one crystallographic axis will not induce polarization along the other two axes. If a crystal is purely ionic, the nonzero diagonal BECs should have values approach their nominal ionic charges. Because the three components of the BECTs of the Sn and S ions are not equal, so we averaged them and gained the values: 3.94 and -1.97 for the Sn and S ions, respectively. These values are close to their respective nominal charges: +4 and -2 , which hints that $\mathrm{SnS}_{2}$ has strong ionic characteristic.

Dielectric constant is intimately related to the resistant ability of materials against the exterior electric field. When the frequency of the electric field is low, nuclei and electrons can be polarized simultaneously, so both contribute to the dielectric constant. The electronic dielectric constant can be calculated according to the definition:

$$
\varepsilon_{\alpha \beta}^{\infty}=\delta_{\alpha \beta}+\left.4 \pi \frac{\partial P_{\alpha}}{\partial E_{\beta}}\right|_{\tau=0},
$$

where $P_{\alpha}$ is the $\alpha$ component of the polarization $P$ and $E_{\beta}$ is the $\beta$ component of the exerted electric field $E$. The total dielectric constant can be deduced from the complicated formula given in Ref. [34].

For a hexagonal crystal, the electronic $\left(\varepsilon_{\alpha \beta}^{\mathrm{e}}\right)$ and ionic $\left(\varepsilon_{\alpha \beta}^{\mathrm{i}}\right)$ dielectric tensors of the consistent ions are diagonal. Both have two independent nontrivial components $\varepsilon_{\perp}=\varepsilon_{x x}=\varepsilon_{y y}$ and $\varepsilon_{\|}=\varepsilon_{z z}$, signifying the components perpendicular and parallel to the $c$ axis, respectively. The calculated electronic dielectric constants of $\mathrm{SnS}_{2}$ are $\varepsilon_{\perp}^{e}=8.80$ and $\varepsilon_{\|}^{\mathrm{e}}=6.44$ and the total dielectric constants are $\varepsilon_{\perp}^{\mathrm{t}}=20.26$ and $\varepsilon_{\|}^{\mathrm{t}}=6.98$, so the ionic dielectric constants are $\varepsilon_{\perp}^{\mathrm{i}}=11.46$ and $\varepsilon_{\|}^{\mathrm{i}}=0.54$. The perpendicular component of the ionic dielectric constants is greater than that of the electrons, but its parallel component is far smaller than that of the latter owing to the weak interaction along the $c$ axis.

\section{Conclusions}

We studied the electronic, elastic and lattice dynamic properties of $\mathrm{SnS}_{2}$ by first-principles calculations. The relaxed lattice constants are in good agreement with the experimental ones. Band structure calculated by the HSE06 functional indicates that $\mathrm{SnS}_{2}$ is an indirect band 
gap semiconductor with a gap of $2.16 \mathrm{eV}$. Analyses on the partial density of states and Mulliken charges reveal that $\mathrm{SnS}_{2}$ is an ionic-covalent compound. $\mathrm{SnS}_{2}$ is mechanically and dynamically stable, but the small values of the elastic constants $C_{44}(12.8 \mathrm{GPa})$ and $C_{13}(16.9 \mathrm{GPa})$ imply that crack is easy to take place along the $c$ axis for the weak van der Waals interaction in this direction. Analyses reveal that there are infrared- and Ramanactive modes at its Brillouin zone center. The frequencies of these modes are computed and their vibrational patters are discussed. Studies of its Born effective charges and dielectric constants uncover that ions have a larger contribution than electrons to the perpendicular dielectric component, but they have very little contribution to the parallel dielectric component, which should originate from the layered structure of $\mathrm{SnS}_{2}$.

\section{References}

[1] J.E. Padilha, H. Peelaers, A. Janotti, C.G. Van de Walle, Phys. Rev. B 90, 205420 (2014).

[2] H. Li, Q. Zhang, C.C.R. Yap, B.K. Tay, T.H.T. Edwin, A. Olivier, D. Baillargeat, $A d v$. Funct. Mater. 22, 1385 (2012).

[3] K. Chang, X. Hai, H. Pang et al., Adv. Mater. 28 10033 (2016).

[4] L. Kou, C. Tang, Y. Zhang, T. Heine, C. Chen, T. Frauenheim, J. Phys. Chem. Lett. 3, 2934 (2012).

[5] W. Du, D. Deng, Z. Han, W. Xiao, C. Bian, X. Qian, Cryst. Eng. Commun. 13, 2071 (2011).

[6] L.A. Burton, D. Colombara, R.D. Abellon, F.C. Grozema, L.M. Peter, T.J. Savenije, G. Dennler, A. Walsh, Chem. Mater. 25, 4908 (2013).

[7] C. Julien, M. Eddrief, I. Samaras, M. Balkanski, Mater. Sci. Eng. B 15, 70 (1992).

[8] A.J. Smith, P.E. Meek, W.Y. Liang, J. Phys. C Solid State Phys. 10, 1321 (1977).

[9] C. Wang, K. Tang, Q. Yang, Y. Qian, Chem. Phys. Lett. 357, 371 (2002).

[10] S.V. Bhatt, M.P. Deshpande, V. Sathe, S.H. Chaki, Solid State Commun. 201, 54 (2015).

[11] P.E. Lippens, M. ElKhalifi, M. Womes, Phys. Status Solidi B 254, 1600194 (2017).
[12] M.Ø. Fils $\varnothing$, E. Eikeland, J. Zhang, S.R. Madsen, B.B. Iversen, Dalton Trans. 45, 3798 (2016).

[13] A. Giberti, A. Gaiardo, B. Fabbri et al., Sensors Actuat. B Chem. 223, 827 (2016).

[14] Y. Xiong, W. Xu, D. Ding, W. Lu, L. Zhu, Z. Zhu, Y. Wang, Q. Xue, J. Hazard. Mater. 341, 159 (2018).

[15] R. Wei, J. Hu, T. Zhou, X. Zhou, J. Liu, J. Li, Acta Mater. 66, 163 (2014).

[16] X. Chia, P. Lazar, Z. Sofer, J. Luxa, M. Pumera, J. Phys. Chem. C 120, 24098 (2016).

[17] X. He, H. Shen, Physica B 407, 1146 (2012).

[18] H. Wang, Y. Gao, G. Liu, RSC Adv. 7, 8098 (2017).

[19] J.M. Gonzalez, I.I. Oleynik, Phys. Rev. B 94, 125443 (2016).

[20] A.V. Bandura, R.A. Evarestov, Surf. Sci. 641, 6 (2015).

[21] H. Chang, E. In, K. Kong, J.O. Lee, Y. Choi, B.H. Ryu, J. Phys. Chem. B 109, 30 (2005).

[22] P. Guo, T. Wang, C. Xia, Y. Jia, Appl. Phys. A 122 684 (2016).

[23] A. Shafique, A. Samad, Y.H. Shin, Phys. Chem. Chem. Phys. 19, 20677 (2017).

[24] M.D. Segall, P.L.D. Lindan, M.J. Probert, C.J. Pickard, P.J. Hasnip, S.J. Clark, M.C. Payne, J. Phys. Condens. Matter 14, 2717 (2002).

[25] J.P. Perdew, K. Burke, M. Ernzerhof, Phys. Rev. Lett. 77, 3865 (1996).

[26] S. Grimme, J. Comput. Chem. 27, 1787 (2006).

[27] B. Pałosz, W. Steurer, H. Schulz, Acta Crystallogr. B 46, 449 (1990).

[28] R.M. Hazen, L.W. Finger, Am. Mineral. 63, 289 (1978).

[29] A. Degrauw, R. Armstrong, A.A. Rahman, J. Ogle, L. Whittaker-Brooks, Mater. Res. Express 4, 094002 (2017).

[30] J. Heyd, G.E. Scuseria, M. Ernzerhof, J. Chem. Phys. 118, 8207 (2003).

[31] Z. Lv, H. Cui, H. Huang, X. Li, H. Wang, G. Ji, J. Alloys Compd. 692, 440 (2017).

[32] S.F. Pugh, Philos. Mag. 45, 833 (1954).

[33] J. Haines, J.M. Leger, G. Bocquillon, Annu. Rev. Mater. Res. 31, 1 (2001).

[34] X. Gonze, C. Lee, Phys. Rev. B 55, 10355 (1997). 